$10-7-2018$

\title{
With-in host Dynamics of L. monocytogenes and Thresholds for Distinct Infection Scenarios
}

\author{
Ashrafur Rahman \\ York University, rahman2@oakland.edu \\ Daniel Munther \\ Cleveland State University, d.munther@csuohio.edu \\ Aamir Fazil \\ National Microbiology Laboratory \\ Ben Smith \\ National Microbiology Laboratory \\ Jianhong Wu \\ York University
}

Follow this and additional works at: https://engagedscholarship.csuohio.edu/scimath_facpub

Part of the Food Microbiology Commons, and the Mathematics Commons

How does access to this work benefit you? Let us know!

\section{Repository Citation}

Rahman, Ashrafur; Munther, Daniel; Fazil, Aamir; Smith, Ben; and Wu, Jianhong, "With-in host Dynamics of L. monocytogenes and Thresholds for Distinct Infection Scenarios" (2018). Mathematics Faculty Publications. 327.

https://engagedscholarship.csuohio.edu/scimath_facpub/327

This Article is brought to you for free and open access by the Mathematics and Statistics Department at EngagedScholarship@CSU. It has been accepted for inclusion in Mathematics Faculty Publications by an authorized administrator of EngagedScholarship@CSU. For more information, please contact library.es@csuohio.edu. 


\title{
With-in host dynamics of $L$. monocytogenes and thresholds for distinct infection scenarios
}

\author{
Ashrafur Rahman Daniel Munther Aamir Fazil Ben Smith Jianhong Wu
}

A R T I C L E I N F O

Keywords:

Food-borne pathogen

Listeriosis

Systemic infection

T-cells

With-in host dynamics

\begin{abstract}
A B S T R A C T
The case fatality and illness rates associated with L. monocytogenes continue to pose a serious public health burden despite the significant efforts and control protocol administered by private and public sectors. Due to the advance in surveillance and improvement in detection methodology, the knowledge of sources, transmission routes, growth potential in food process units and storage, effect of $\mathrm{pH}$ and temperature are well understood. However, the with-in host growth and transmission mechanisms of $L$. monocytogenes, particularly within the human host, remain unclear, largely due to the limited access to scientific experimentation on the human population. In order to provide insight towards the human immune response to the infection caused by L. monocytogenes, we develop a with-in host mathematical model. The model explains, in terms of biological parameters, the states of asymptomatic infection, mild infection and systemic infection leading to listeriosis. The activation and proliferation of T-cells are found to be critical for the susceptibility of the infection. Utilizing stability analysis and numerical simulation, the ranges of the critical parameters relative to infection states are established. Bifurcation analysis shows the impact of the differences of these parameters on the dynamics of the model. Finally, we present model applications in regards to predicting the risk potential of listeriosis relative to the susceptible human population.
\end{abstract}

\section{Introduction}

L. monocytogenes has played a key role in immuno-logical research and public health over the nine decades since its discovery by Murray, Webb, and Swann in 1924 (Vázquez-boland et al., 2001). Because of recent outbreaks and potential case fatality rate, the gram positive bacterium continues to be a concern for public health and the economy (Buchanan et al., 2017; CDC, 2018; Farber et al., 1996; Foodnet, 2017; PHAC, 2018; Pouillot et al., 2016). With $20-30 \%$ casualty rate and $92-99 \%$ hospitalization rate, L. monocytogenes stands among the top deadly food pathogens (Buchanan et al., 2017; CDC, 2018; Mead et al., 1999). Pregnant women, elderly people and immuno-compromised individuals (e.g. infected with chronic diseases) constitute more than $90 \%$ of those susceptible to L. monocytogenes infections (CDC, 2018). Understanding the key infection mechanisms of L. monocytogenes is necessary for pri- oritizing prevention methodologies and reducing the infection burden.

Much has been discovered of the molecular mechanisms of L. monocytogenes including how this pathogen moves cell-to-cell, survives and proliferates with-in the cells, and escapes the destruction inside phagosomes (Artis, 2008; Cossart, 2011; Vázquezboland et al., 2001). Having the virulence factor, hemolysin gene, L. monocytogenes is a model bacteria, illuminating the molecular mechanisms of intracellular parasitism. In addition, L. monocytogenes studies have provided fundamental understanding of the cellular immune response: the role of activated macrophages for cellmediated immunity and the elimination of intracellular pathogens (Artis, 2008; Vázquez-boland et al., 2001).

On the larger scale, potential impact of outbreaks have also been studied using microbial risk assessment tools (Buchanan et al., 2017; Falk et al., 2016). Substantial research has been dedicated to elucidate dose-response relationships and to identify a safe contamination dose for L. monocytogenes (Buchanan et al., 2017; FAO/WHO, 2004; Farber et al., 1996; Haas et al., 1999; Pouillot et al., 2016). Taking a slightly different perspective, Rahman 
et al. recently proposed a mathematical model to describe the infection pathway (from ingestion to colonization of the small intestine) in guinea pigs. This is the first study to quantitatively link $L$. monocytogenes dose-response outcomes to the pathogenhost interaction in the gastro-intestinal path of the infected host (Rahman et al., 2016). These studies together with feeding trials (Farber et al., 1996; Roulo et al., 2014; Smith et al., 2008) provide tools to estimate the risk associated with contaminated food products on public health (Buchanan et al., 2017; Farber et al., 1996; Pouillot et al., 2016).

Molecular research in conjunction with outbreak findings portray the gravity of Listeriosis. In particular, the quantification of the pathogen growth under a potentially wide range of varying conditions relative to susceptible hosts is vital for identifying the risk of infection. Understanding the with-in host growth mechanism and the pathogen-host interaction would be helpful to reduce the infection burden. The growth kinetics of $L$. monocytogenes have been extensively studied in multiple media with control environments (Blanco-Lizarazo et al., 2016; Schvartzman et al., 2014). However, the with-in host growth and host-pathogen interactions that translate the success or failure of human infection are not yet clearly understood. Due to the limits associated to direct scientific experimentation on the human population, studies mostly focus on animal exposure. Many fundamental questions associated with risk factors thus remain open with great uncertainty. For instance, does the L. monocytogenes start growing right away after ingestion? Are there any delays involved in bacterial replication? What are the key defense elements of the host and how can one quantify their relative effectiveness against infection? When and how fast is the adaptive immune system activated in response to infection? What are the variations or distributions of immune potentiality across the population? Given the importance of these questions in regard to accurately quantifying infection risk and the limitations of human experimentation, we propose that mathematical modeling can play key role in addressing such issues.

In particular, we develop a mathematical model of with-in host dynamics of $L$. monocytogenes describing the host-pathogen interactions. We define the infection as a function of growth and kill factors and highlight the parameter region for which $L$. monocytogenes can pose severe infection, be wiped out or remain in the host for a long period without causing infections. The rest of this paper is organized as follows: Section 2 develops the dynamical model demonstrating the host-pathogen interaction; Section 3 describes the basic analysis of the model; Section 4 deals with stability analysis and finds the thresholds for different infection scenarios; Section 5 illustrates the possible outcomes of the model and demonstrate the sensitivity and significance of the key parameters. Finally, Section 6 discusses the outcomes, outlines the applications of the model and identifies the caveats and possible developments of the model.

\section{Model: systemic infection}

Listeria monocytogenes upon consumption travels from the mouth to the stomach of the host. The stomach is very acidic and hostile for any organism. However, if large number of L. monocytogenes is ingested a small portion of them could survive in the stomach and move to the small intestine. The small intestine enriched with nutrition and a low acid level is relatively a less stressful environment for a pathogen (Rahman et al., 2016). The bacteria can replicate and establish a niche in the small intestine. The bacteria further move and cross the intestinal wall through the tip of the villi (Ribet and Cossart, 2015) and disseminate into liver, spleen, etc through the blood and lymphatic systems. The bacteria can grow both in the small intestine as well as in key organs (e.g. spleen) after crossing the intestinal wall. For simplicity, we consider the state and dynamics of the L. monocytogenes in the small intestine and in the lamina propria. The dynamics of the bacteria in the other organs/sites would be similar to that of the lamina propria. The infection or disease outcomes with regards to $L$. monocytogenes can be translated from the dynamics of the bacteria at these two sites. The dynamics of L. monocytogenes in the stomach is well described in Rahman et al. (2016). Here we consider the dynamics of the bacteria that survive the stomach and reach the small intestine.

The growth of the pathogenic bacteria is inhibited by the commensal bacteria in the small intestine (Artis, 2008; Stecher and Hardt, 2011). The bacteria are killed or trapped by the innate immune cells, e.g. dendritic cells and macrophages, and by the intestinal molecules as detailed in Rahman et al. (2016). If infection persists the adaptive immune cell, particularly, T-cell proliferates and eliminates the pathogens (Pamer, 2004; Seaman et al., 2000). The dynamics of $L$. monocytogenes in the small intestine and the justification of the mathematical forms are well established in a previous study (Rahman et al., 2016). This study assumed that: (1) The growth of the bacteria is limited by commensals and the intestinal environment, that is, the bacteria cannot grow beyond the carrying capacity ( $K \mathrm{CFU}$ ) of the small intestine and follows the logistic growth; (2) In the small intestine, L. monocytogenes can reproduce and may be killed by immune cells and other molecules (e.g. bile). The authors further assume that the population of immune cells and other molecules that kill L. monocytogenes collectively remain constant. Following scientific experiments and epidemiological evidences, in this study, we further assume that (3) The T-cell is responsible for eliminating the pathogen from the host and takes the major role in eliminating the bacteria followed by the mass action principle (Hethcote, 2000); (4) The T-cell remains active in the presence of bacteria, proliferates at the rate of $q$ per hour per bacterium and die at a rate of $\mu$ per hour. (5) $L$. monocytogenes disseminates to the lamina propria from the small intestine at the rate of $m$. Following these assumptions, the withinhost dynamics can be described by the following system of differential equations:

$$
\begin{aligned}
L_{i}^{\prime} & =r_{i} L_{i}\left(1-\frac{L_{i}}{K_{i}}\right)-\frac{C L_{i}}{1+\alpha L_{i}}-\beta_{T} T L_{i}-m L_{i}, \\
L_{p}^{\prime} & =m L_{i}-\beta_{T} T L_{p}+r_{p} L_{p}\left(1-\frac{L_{p}}{K_{p}}\right), \\
T^{\prime} & =q T\left(L_{i}+L_{p}\right)-\mu T .
\end{aligned}
$$

The variables and parameters of the model (2.1) are described in Table 1.

\section{Model analysis}

Model (2.1) has two states of $L$. monocytogenes and one immune component of the infected host. Our primary goal is to investigate under what situations one or both states of the pathogen could survive and cause infection. The strength of the immune response is important to prevent infection. Understanding the long term behaviour of the model solution would provide the key information of the model parameters for the persistence of infection. By elementary arguments, we can show that the solutions of the model (2.1) fall in a biologically plausible region using the text of Smith and Waltman (1995). In order to find the long term behaviour of the model, we find the equilibria of the model and analyse the stability of these equilibria.

The model (2.1) has five equilibria, namely $E_{0}=(0,0,0), E_{P}=$ $\left(0, K_{p}, 0\right), E_{P T}=\left(0, \frac{\mu}{q}, \frac{r_{p}}{\beta_{T}}\left(1-\frac{\mu}{q K_{p}}\right)\right), \quad E_{I P}=(-,-, 0) \quad$ and $E_{*}=(*, *, *)$. The first three equilibria are explicitly defined. The conditions of the existence of $E_{I P}$ and $E_{*}$ are described below. 
Table 1

Description of variables and parameters of model (2.1).

\begin{tabular}{ll}
\hline Parameter & Description \\
\hline$L_{i}(t)$ & L. monocytogenes population in intestine \\
$L_{p}(t)$ & L. monocytogenes population in lanina propria \\
$T(t)$ & L. monocytogenes specific T-cell \\
$r_{i}$ & growth rate of $L$. monocytogenes in the small intestine \\
$r_{p}$ & growth rate of $L$. monocytogenes in the lamina propria \\
$K_{i}$ & carrying capacity of $L$. monocytogenes in the sull intestine \\
$K_{p}$ & carrying capacity of $L$. monocytogenes in the lamina propria \\
$C$ & killing rate of $L$. monocytogenes in the small intestine by intestinal molecule/cell \\
$\beta_{T}$ & killing rate of $L$. monocytogenes by T-cell \\
$\alpha$ & saturating constant \\
$m$ & dissemination rate of $L$. monocytogenes from the small intestine \\
$q$ & proliferation rate of T-cell \\
$\mu$ & death rate of T-cell
\end{tabular}

\subsection{Existence of $\mathrm{E}_{1 \mathrm{P}}$}

Regarding the equilibrium $E_{I P}=(-,-, 0)$, we have the following equations:

$$
\begin{array}{r}
r_{i}\left(1-\frac{L_{i}}{K_{i}}\right)-\frac{C}{1+\alpha L_{i}}-m=0, \\
m L_{i}+r_{p} L_{p}\left(1-\frac{L_{p}}{K_{p}}\right)=0 .
\end{array}
$$

By simplifying the first and second equations of (3.1), we obtain

$\alpha r_{i} L_{i}^{2}+\left(r_{i}+\alpha m K_{i}-\alpha r_{i} K_{i}\right) L_{i}+\left(C+m-r_{i}\right) K_{i}=0$.

and

$r_{p} L_{p}^{2}-K_{p} r_{p} L_{p}-K_{p} m L_{i}=0$.

Therefore,

$L_{p}=\frac{K_{p} r_{p} \pm \sqrt{\left(K_{p} r_{p}\right)^{2}+4 r_{p} K_{p} m L_{i}}}{2 r_{p}}$.

respectively. It shows from (3.3) that for each positive $L_{i}$ there is a unique positive $L_{p}$. Combining (3.2) and (3.3), we have the following Theorem regarding the existence of $E_{I P}$.

\section{Theorem 3.1.}

1. If $c+m<r_{i}$, then there is a unique $E_{I P}$.

2. If $c+m>r_{i}$, then

(a) if $r_{i}+\alpha m K_{i}<\alpha r_{i} K_{i}$, then there exists two $E_{I P}$, and

(b) if $r_{i}+\alpha m K_{i}>\alpha r_{i} K_{i}$, then $E_{I P}$ does not exist.

\subsection{Existence of $\mathrm{E}_{*}$}

At the equilibrium $E_{*}=(*, *, *)$, we have the following equations:

$$
\begin{array}{r}
r_{i}\left(1-\frac{L_{i}}{K_{i}}\right)-\frac{C}{1+\alpha L_{i}}-\beta_{T} T-m=0, \\
m L_{i}-\beta_{T} T L_{p}+r_{p} L_{p}\left(1-\frac{L_{p}}{K_{p}}\right)=0, \\
q\left(L_{i}+L_{p}\right)-\mu=0 .
\end{array}
$$

From the 3 rd equation of (3.4), we have

$L_{p}=\frac{\mu}{q}-L_{i}$ and $0<L_{i}, L_{p}<\frac{\mu}{q}$.

Replacing $L_{p}$ from the 1 st and 2 nd equations of (3.4), we obtain

$\beta_{T} T=m L_{i}+r_{p}\left(1-\frac{L_{p}}{K_{p}}\right)$

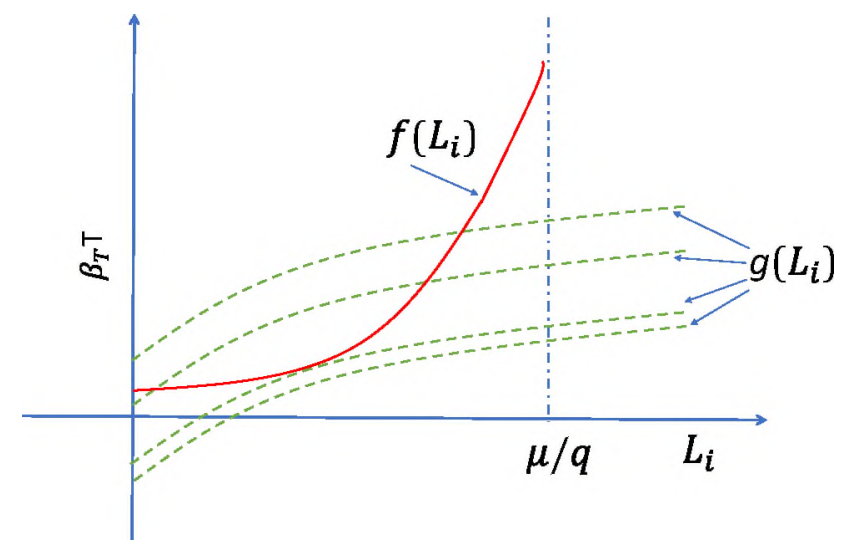

Fig. 1. Schematic illustration to show when we should expect the existence of $E_{x}$. Model (2.1) could have none, one or two equilibria subject to the intersections of the two curves given by $f\left(L_{i}\right)$ and $g\left(L_{i}\right)$.

$$
=\frac{m L_{i}}{\frac{\mu}{q}-L_{i}}+r_{p}\left(1-\frac{\frac{\mu}{q}-L_{i}}{K_{p}}\right) \equiv f\left(L_{i}\right)
$$

and

$\beta_{T} T=r_{i}\left(1-\frac{L_{i}}{K_{i}}\right)-\frac{c}{1+\alpha L_{i}}-m \equiv g\left(L_{i}\right)$.

The positive equilibrium $E_{*}$ exists if the curves associated with (3.6) and (3.7) intersect for $L_{i} \in\left[0, \frac{\mu}{q}\right]$ as depicted in Fig. 1. It shows that we can expect at most two co-existence equilibria, $E_{*}$. Further exploration of the existence of $E_{*}$ is given in Section 5.3.

With this complete classification about the existence of equilibria of the model (2.1), we can determine the stability conditions of these equilibria.

\section{Local stability}

We can gain insight about the long term behaviour of the model solution by analysing the stability of the equilibria. In order to determine the stability, we linearize model (2.1) and evaluate the eigenvalues of the linearized system. The Jacobian of model (2.1) is given by

$J=\left(\begin{array}{lll}r_{i}-\frac{2 r L_{i}}{K_{i}}-\frac{c}{1+\alpha L_{i}}+\frac{\alpha L_{i}}{\left(1+\alpha L_{i}\right)^{2}}-\beta_{T} T-m & 0 & -\beta_{T} L_{i} \\ m & -\beta_{T} T+r_{p}-\frac{2 r_{p} L_{p}}{K_{p}} & -\beta_{T} L_{p} \\ q T & q T & q\left(L_{i}+L_{p}\right)-\mu\end{array}\right)$

In the following, we evaluate the Jacobian at the equilibria of model (2.1) and determine their stability. 
Evaluating $J$ at $E_{0}$, we get

$J\left(E_{0}\right)=\left(\begin{array}{ccc}r_{i}-m-C & 0 & 0 \\ m & r_{p} & 0 \\ 0 & 0 & -\mu\end{array}\right)$.

The eigenvalues of $J\left(E_{0}\right)$ are given by $r_{i}-m-c, r_{p}$ and $-\mu$. Since one of the eigenvalues of $J\left(E_{0}\right), r_{p}$, is positive, the equilibrium $E_{0}$ is unstable. This gives us the following result.

Theorem 4.1. The trivial equilibrium $E_{0}$ is always unstable.

\subsection{Stability of $\mathrm{E}_{\mathrm{P}}$}

Evaluating $J$ at $E_{P}$ we obtain

$J\left(E_{P}\right)=\left(\begin{array}{ccc}r_{i}-m-C & 0 & 0 \\ m & -r_{p} & -\beta_{T} K_{p} \\ 0 & 0 & q K_{p}-\mu\end{array}\right)$.

The eigenvalues of $J\left(E_{P}\right)$ are given by $-r_{p}, q K_{p}-\mu$ and $r_{i}-m-C$. The stability of $J\left(E_{P}\right)$ depends on the signs of these eigenvalues as described in the following Theorem.

Theorem 4.2. The equilibrium $E_{p}$ is locally stable if $q K_{p}<\mu$ and $r_{i}<$ $m+C$. Otherwise, it is unstable.

We note that if $E_{P}$ is stable (i.e. $q K_{p}-\mu<0$ ) then $E_{P T}$ does not exist. That is these two equilibria could not be stable simultaneously.

\subsection{Stability of $\mathrm{E}_{\mathrm{PI}}$}

The Jacobian at $E_{P T}$ is given by

$$
\begin{aligned}
J\left(E_{P T}\right) & =\left(\begin{array}{ccc}
r_{i}-C-m-\beta_{T} T & 0 & 0 \\
m & -\beta_{T} T+r_{p}-2 \frac{r_{p} L_{p}}{K_{p}} & -\beta_{T} L_{p} \\
q T & q T & q\left(L_{i}+L_{p}\right)-\mu
\end{array}\right) \\
& =\left(\begin{array}{ccc}
r_{i}-C-m-r_{p}\left(1-\frac{\mu}{q K_{p}}\right) & 0 & 0 \\
m & -r_{p}\left(1-\frac{\mu}{q K_{p}}\right)+r_{p}-2 \frac{\mu r_{p}}{q K_{p}} & -\beta_{T} \frac{\mu}{q} \\
q \frac{r_{p}}{\beta_{T}}\left(1-\frac{\mu}{q K_{p}}\right) & q \frac{r_{p}}{\beta_{T}}\left(1-\frac{\mu}{q K_{p}}\right) & 0
\end{array}\right) .
\end{aligned}
$$

Clearly, one of the eigenvalues of $J\left(E_{P T}\right)$ is $\lambda_{1}=r_{i}-C-m-r_{p}(1-$ $\frac{\mu}{q K_{p}}$ ), the other two are found to be negative by testing the determinant and trace of the right lower block of $J\left(E_{P T}\right)$. Thus the stability of $E_{P T}$ solely depends on $\lambda_{1}$ and we have following result.

Theorem 4.3. The equilibrium $E_{P T}$ is locally stable if

$r_{i}<C+m+r_{p}\left(1-\frac{\mu}{q K_{p}}\right)$.

Otherwise, it is unstable.

\subsection{Stability of $\mathrm{E}_{\mathrm{lP}}$}

The Jacobian at $E_{I P}$ is given by

$J\left(E_{I P}\right)=\left(\begin{array}{ccc}\lambda_{1} & 0 & -\beta_{T} L_{i} \\ m & \lambda_{2} & -\beta_{T} L_{p} \\ 0 & 0 & \lambda_{3}\end{array}\right)$,

where

$$
\begin{aligned}
\lambda_{1} & =r_{i}-\frac{2 r_{i} L_{i}}{K_{i}}-\frac{C}{1+\alpha L_{i}}+\frac{\alpha C L_{i}}{\left(1+\alpha L_{i}\right)^{2}}-m, \\
& =\left(\frac{\alpha C}{\left(1+\alpha L_{i}\right)^{2}}-\frac{r_{i}}{K_{i}}\right) L_{i}, \\
& \leq\left(\alpha C-\frac{r_{i}}{K_{i}}\right) L_{i} .
\end{aligned}
$$

$$
\begin{aligned}
\lambda_{2} & =r_{p}\left(1-\frac{2 L_{p}}{K_{p}}\right), \\
& =r_{p}\left(1-\frac{L_{p}}{K_{p}}\right)-\frac{r_{p} L_{p}}{K_{p}}, \\
& =-\frac{m L_{i}}{L_{p}}-\frac{r_{p} L_{p}}{K_{p}}<0,
\end{aligned}
$$

and

$\lambda_{3}=q\left(L_{i}+L_{p}\right)-\mu$,

where $L_{j}$ and $L_{p}$ are given by Eq. (3.1). Also, we have used Eq. (3.1) in simplifying $\lambda_{1}, \lambda_{2}$ and $\lambda_{3}$. Note that $q$ is independent of $L_{i}$ and $L_{p}$ components of $E_{I P}$. Thus $\lambda_{3}$ can be made negative for a small $q$ so that $q\left(L_{i}+L_{p}\right)<\mu$. Moreover, when $\alpha$ is zero then $\lambda_{1}$ becomes negative. In the later case, $E_{I P}$ is unique (Theorem 3.1).

Theorem 4.4. If $\alpha<\frac{r_{i}}{C K_{i}}$ and $L_{i}+L_{p}<\frac{\mu}{q}$, the equilibrium $E_{I P}$ is stable.

\subsection{Stability of $\mathrm{E}_{*}$}

Now we determine the stability of $E_{*}$. Evaluating $J$ at $E_{*}$, we obtain

$J\left(E_{*}\right)=\left(\begin{array}{ccc}a & 0 & -\beta_{T} L_{i} \\ m & b & -\beta_{T} L_{p} \\ q T & q T & 0\end{array}\right)$,

where

$a=-\frac{r_{i} L_{i}}{K_{i}}+\frac{\alpha C L_{i}}{\left(1+\alpha L_{i}\right)^{2}}$,

and

$b=-\frac{m L_{i}}{L_{p}}-\frac{r_{p} L_{p}}{K_{p}}$

The characteristic polynomial of $J\left(E_{*}\right)$ is given by

$$
\begin{aligned}
p(\lambda)= & \lambda^{3}+\alpha_{1} \lambda^{2}+\alpha_{2} \lambda+\alpha_{3}, \\
= & \lambda^{3}-(a+b) \lambda^{2}+\left(q \beta_{T} L_{i} T+q \beta_{T} L_{p} T+a b\right) \lambda \\
& +q m \beta_{T} L_{i} T-q \beta_{T} T\left(b L_{i}+a L_{p}\right), \\
= & \lambda^{3}-(a+b) \lambda^{2}+\left(\mu \beta_{T} T+a b\right) \lambda+q m \beta_{T} L_{i} T \\
& -q \beta_{T} T\left(b L_{i}+a L_{p}\right) .
\end{aligned}
$$

For the stability of $E_{*}$, the following Hurwitz determinants must be positive.

$\Delta_{1}=\alpha_{1}$,

$\Delta_{2}=\alpha_{1} \alpha_{2}-\alpha_{3}$,

$\Delta_{3}=\alpha_{3} \Delta_{2}$.

We have,

$$
\begin{aligned}
\alpha_{1} & =-(a+b), \\
& =\frac{r_{i} L_{i}}{K_{i}}-\frac{\alpha C L_{i}}{\left(1+\alpha L_{i}\right)^{2}}+\frac{m L_{i}}{L_{p}}+\frac{r_{p} L_{p}}{K_{p}} . \\
\alpha_{2} & =\mu \beta_{T} T+a b, \\
& =\mu \beta_{T} T+\left(-\frac{r_{i} L_{i}}{K_{i}}+\frac{\alpha C L_{i}}{\left(1+\alpha L_{i}\right)^{2}}\right)\left(-\frac{m L_{i}}{L_{p}}-\frac{r_{p} L_{p}}{K_{p}}\right), \\
& =\mu \beta_{T} T+\left(\frac{r_{i} L_{i}}{K_{i}}-\frac{\alpha C L_{i}}{\left(1+\alpha L_{i}\right)^{2}}\right)\left(\frac{m L_{i}}{L_{p}}+\frac{r_{p} L_{p}}{K_{p}}\right) .
\end{aligned}
$$




$$
\begin{aligned}
\alpha_{3} & =q \beta_{T} T\left(m L_{i}+L_{i}\left(\frac{m L_{i}}{L_{p}}+\frac{r_{p} L_{p}}{K_{p}}\right)-L_{p}\left(-\frac{r_{i} L_{i}}{K_{i}}+\frac{\alpha C L_{i}}{\left(1+\alpha L_{i}\right)^{2}}\right)\right), \\
& =q \beta_{T} T\left(m L_{i}+L_{i}\left(\frac{m L_{i}}{L_{p}}+\frac{r_{p} L_{p}}{K_{p}}\right)+L_{p}\left(\frac{r_{i} L_{i}}{K_{i}}-\frac{\alpha C L_{i}}{\left(1+\alpha L_{i}\right)^{2}}\right)\right),
\end{aligned}
$$

and

$$
\begin{aligned}
\Delta_{2} & =\alpha_{1} \alpha_{2}-\alpha_{3}, \\
& =\left(L_{i} X+Y\right)\left(\mu \beta_{T} T+L_{i} X Y\right)-q \beta_{T} L_{i} T\left(m+Y+L_{p} X\right),
\end{aligned}
$$

where

$X=-\frac{a}{L_{i}}$ and $Y=-b$.

Expanding (4.9), we have

$$
\begin{aligned}
\Delta_{2}= & T \beta_{T} L_{i} X\left(\mu-q L_{p}\right)+T \beta_{T} Y\left(\mu-q L_{i}\right) \\
& +L_{i}\left(L_{i} X^{2} Y+L_{i} X Y^{2}-q m T \beta_{T}\right) .
\end{aligned}
$$

At the equilibrium $E_{*}, 0<L_{i}, L_{p}<\frac{\mu}{q}$. If $\alpha=0, X>0$. It shows that if $\alpha=0$ then $\alpha_{1}, \alpha_{2}$ and $\alpha_{3}$ become positive, and in addition if $q=0$ then $\Lambda_{2}$ as well as all the Hurwitz determinants become positive. It shows that for small $q$ and $\alpha$ there is a stable $E_{*}$ of model (2.1). This is demonstrated in Fig. 3 in the numerical simulation section.

Theorem 4.5. The positive equilibrium $E_{*}$ is locally stable, at least, for some small $q$ and $\alpha$.

The stability analysis of the equilibria of the model is now complete. This analysis provides us with key parameters that dominate the dynamics of the model solution. In the stability conditions, for different equilibria formulated in Theorems (4.1)-(4.5), we have identified a number of thresholds. These thresholds determine when the disease conditions could alter from one state to other. The thresholds also reflect the sensitivity of the parameters. The analytical results will guide us to explore quantitative analysis given in the following section.

\section{Numerical simulation}

The analytical results indicate that 4 out of 5 possible equilibria of the model (2.1) could become stable under certain conditions that solely depend on the model parameters. The only equilibrium that is always unstable regardless of the parameter choice (Theorem 4.1) is $E_{0}$. In this section, we simulate the model to confirm the analytic results and demonstrate the significance of the parameters toward the application of our model for characterizing the potential for human infections.

\subsection{Parameter selection}

Model (2.1) has several parameters, some of which can be found in the literature (Augustin et al., 2005; Lecuit, 2001; Rahman et al., 2016), but the rest can only be estimated within certain biological ranges. Augustine et al. reviewed extensive growth model of L. monocytogenes in multiple media, cheese, meat products, seafood, microbiological media, liquid dairy product, and found the average growth rate of $0.863 \pm 0.43 \mathrm{~h}^{-1}$, varying from 0.24-1.20 (Augustin et al., 2005). The carrying capacity $K_{i}$ or $K_{p}$ is the maximum bacterial load expected for the host's small intestine and lamnia propria, respectively. Animal experiments found that $L$. monocytogenes can grow up to $3.8 \times 10^{6} \mathrm{CFU}$ in the small intestine, lever, spleen and messenteric lymph node of guinea pigs (Lecuit, 2001). Since human organs are 10 to 100 fold larger than the model animals, we consider the mean carrying capacity of $L$. monocytogenes in human organs to be $1 \times 10^{8}$ CFU. The saturation factor $\alpha$ is sensitive to initial population size and could vary $10^{-3}-10^{-10}$ (Rahman et al., 2016), we consider the mean of $\alpha$ is $10^{-7} \mathrm{CFU}^{-1}$. The killing parameter $C$ associated with bacterial death in the small intestine could vary from 0.03-1.58 (Banfi et al., 1986), we consider $C=0.2 \mathrm{~h}^{-1}$ and carry out a bifurcation analysis to observe the significance of $C$ on the model outcomes. The T-cell death usually occurs after infection is over (Crispe, 1999). Following Gorman et al. (2014), Seaman et al. (2000) and Stafford et al. (2000), we deduce that the death rate of T-cells is $\mu=0.01 \mathrm{~h}^{-1}$.

Since the model parameters (Table 2) are from multiple sources that involve uncertainty, we carry out the bifurcation analysis to demonstrate the impact of differences of the parameter values on the model outcomes. Evidences in outbreak data (CDC, 2018; FAO/WHO, 2004; on Immunization (NACI), 2013) demonstrate that certain groups of people are highly susceptible to L. monocytogenes. e.g. pregnant women, elderly people and individuals with a weak immune system. These suggest that the parameters associated with immune response are important for Listeriosis and worth further exploration. To demonstrate the significance of immune response with regards to L. monocytogenes infection, initially, we keep all the parameters at their base value and vary $q$, the proliferation rate of the T-cells.

\subsection{Model simulation for long term behaviour}

With a weak immune response $\left(q=10^{-11}\right)$ the model demonstrates that the L. monocytogenes can survive both in the small intestine and in the lamina propria and reach the steady state as depicted in Fig. 2. Note that the T-cell population does not grow or increase in the presence of pathogens because of the low proliferation rate. This could be regarded as a critically immuno-deficient case.

By increasing $q$ by an order of 10 , the model indicates that immune cells proliferate and remain present to fight the pathogen. The T-cells could not eliminate the pathogens, but could reduce the pathogen load to a degree. The T-cell population and pathogens at both locations reach the steady state in a few days as shown in Fig. 3.

We increase the value of $q$ further, by an order of 10 , to $10^{-9}$. In this case, the T-cell population can eliminate the pathogen from the small intestine. That is, $L_{i}$ goes to zero as time increases. However, the pathogen in the lamina propria $\left(L_{p}\right)$ still survives at a low level. The T-cells and $L_{p}$ reach the steady state over time. Before being eliminated the $L$. monocytogenes population in the small intestine, $L_{i}$, goes up until the T-cell population becomes large and dominates the bacteria, which can be seen in the 3D simulation (refer to Fig. 4).

By increasing $q$ to the order of $10^{-6}$ we find that $L_{p}$ oscillates with T-cell population, but, does not follow a limit cycle as Theorem 4.3 rules out the possibility of Hopf bifurcation. It is worth noting that the two of the eigenvalues of the Jacobian at the equilibrium $E_{P T}$ are always negative- no complex eigenvalues are associated with it. The state variables converge to the steady state very slowly as shown in Fig. 5.

In order to demonstrate the case when the equilibrium $E_{P}$ is stable, we reduce $q$ to $10^{-11}$ so that the T-cells are less active and we increase $C$, the innate immune pressure, to eliminate $L_{i}$, the pathogen in the small intestine. With this choice of parameters, $L_{p}$ survives and approaches the steady state while $L_{i}$ and $T$ die out as can be seen in Fig. 6. This case seems to be unrealistic as T-cells should proliferate in the presence of pathogens.

\subsection{Bifurcation analysis}

In the previous subsection, we demonstrated how the T-cell proliferation rate $q$ can alter the disease dynamics. Now, we consider key parameters for the bifurcation analysis and observe how 

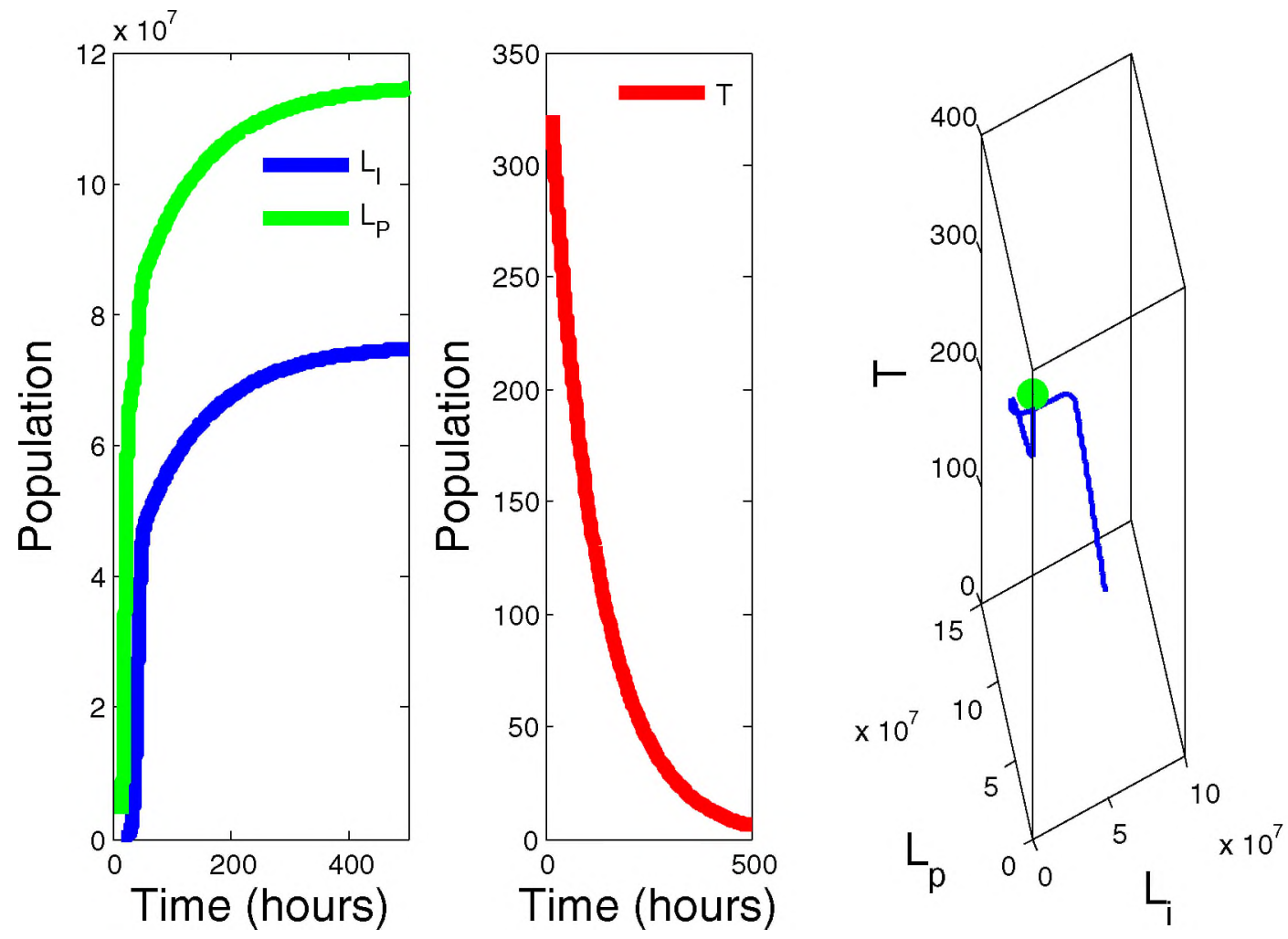

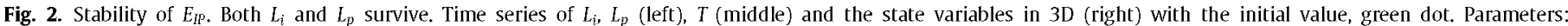

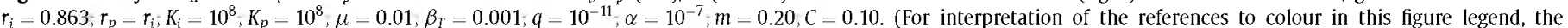
reader is referred to the web version of this article.)
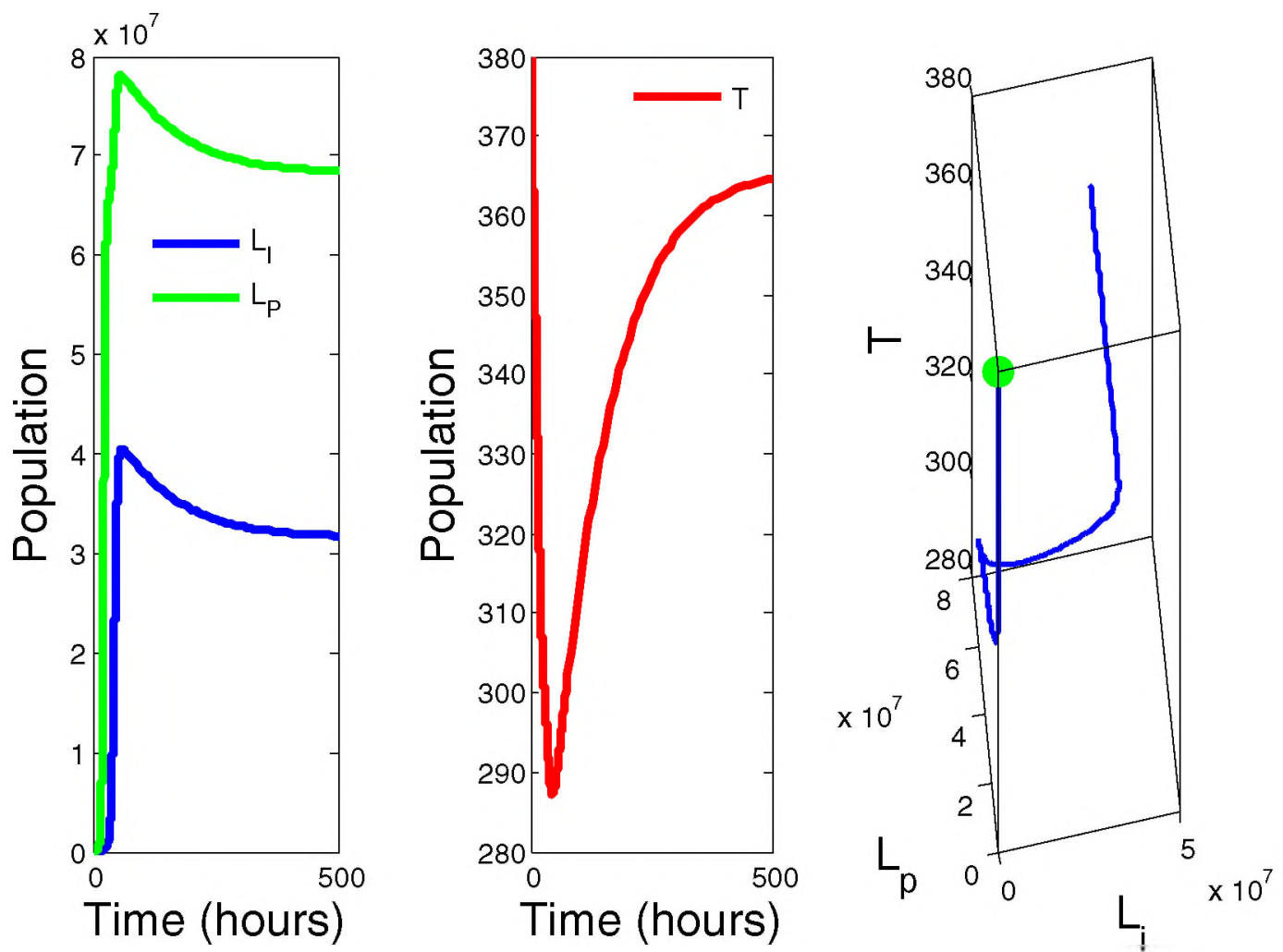

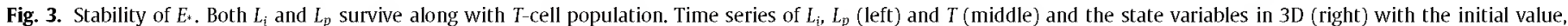

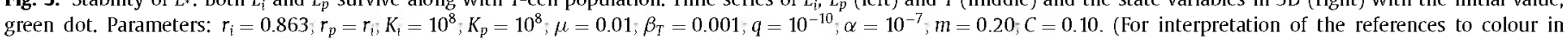
this figure legend, the reader is referred to the web version of this article.) 

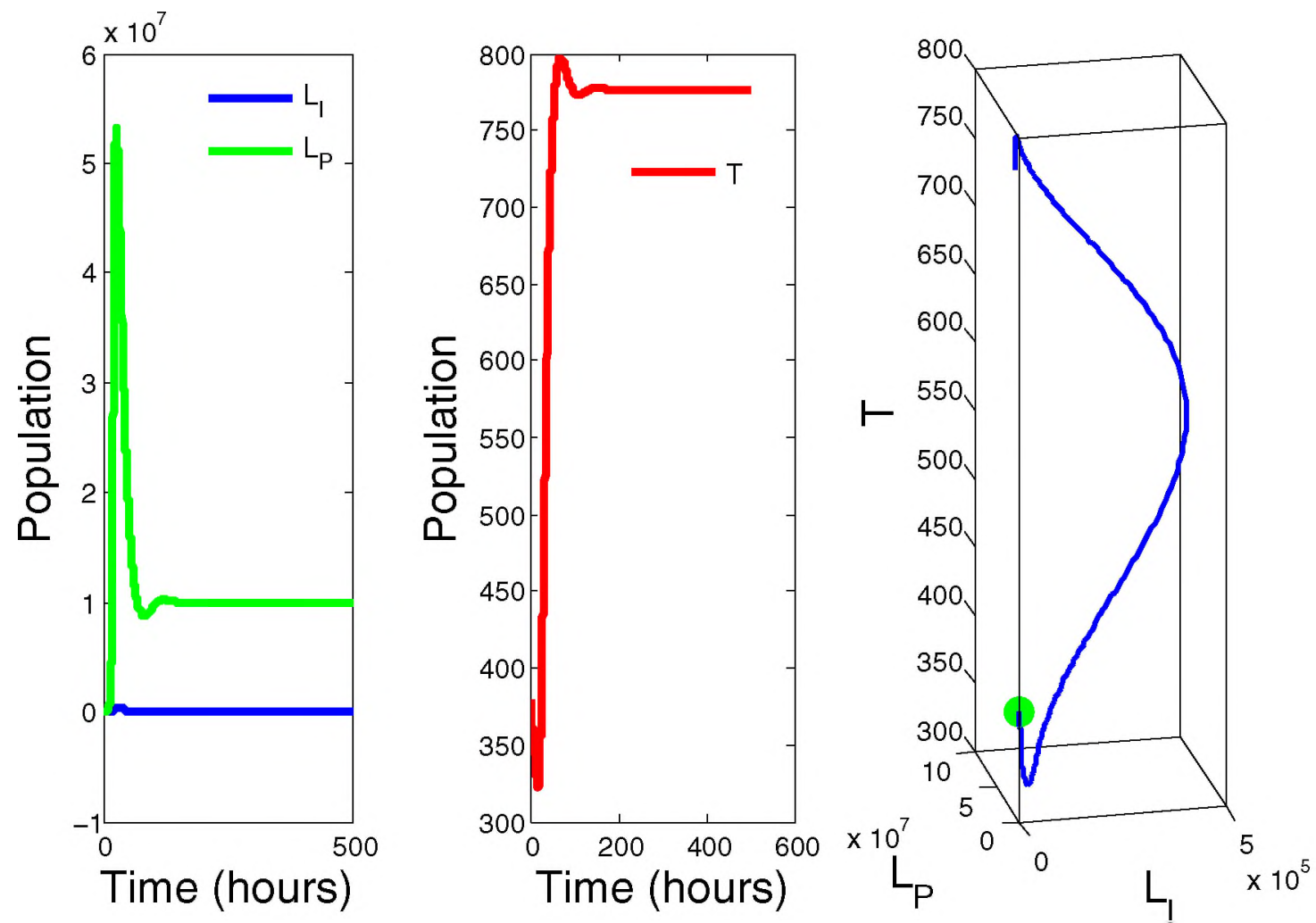

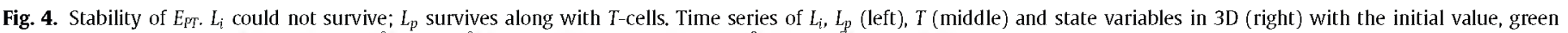

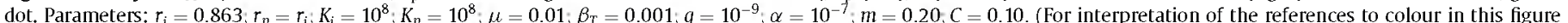
legend, the reader is referred to the web version of this article.)
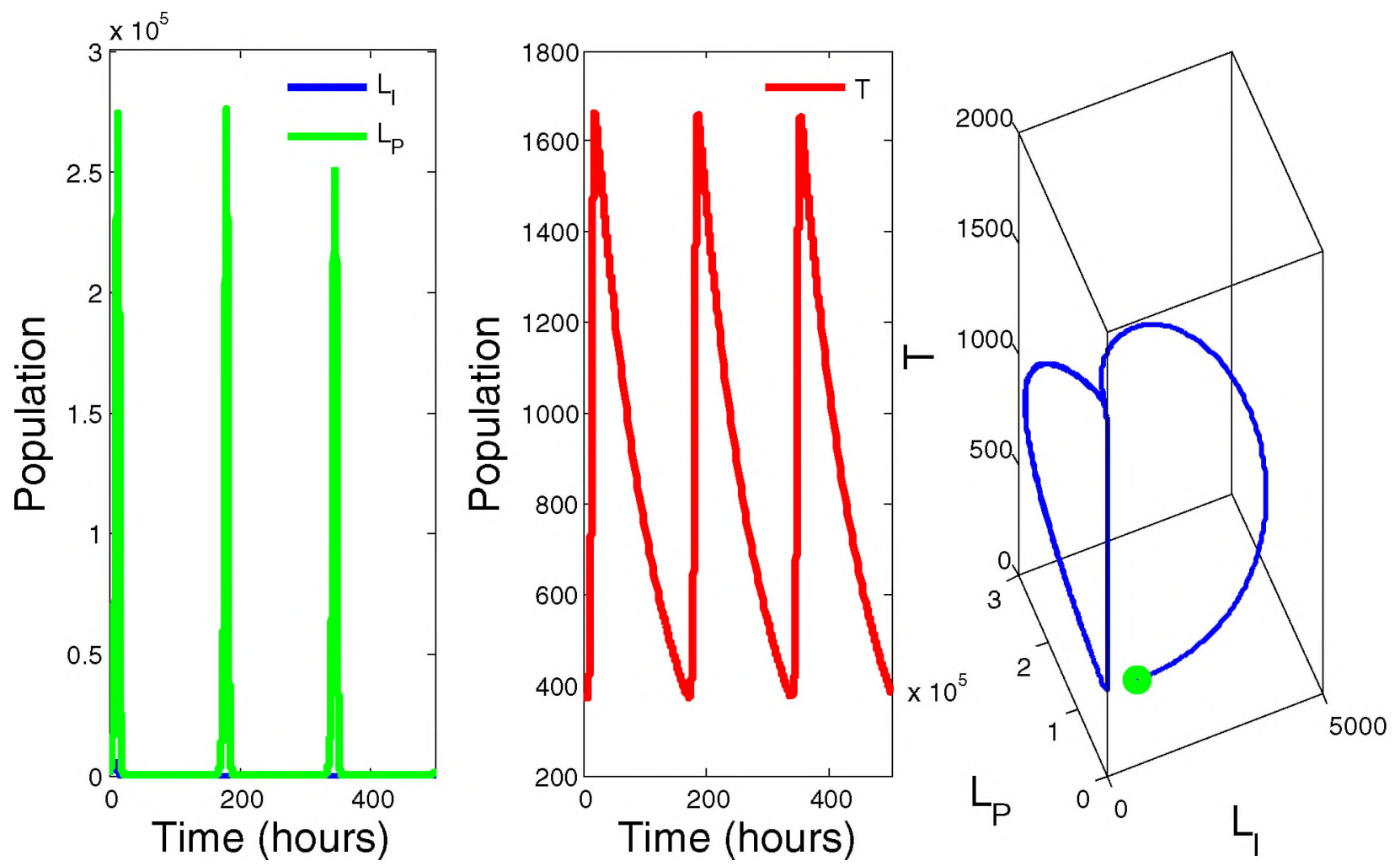

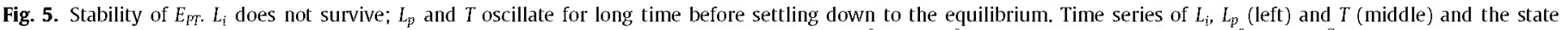

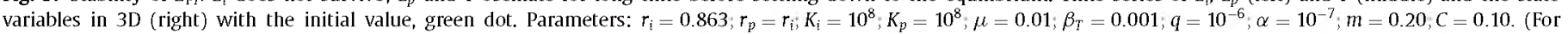
interpretation of the references to colour in this figure legend, the reader is referred to the web version of this article.) 
Table 2

The baseline values of the model parameters.

\begin{tabular}{lllll}
\hline Parameter & Description & Baseline value & Range & References \\
\hline$r_{i}, r_{p}$ & growth rate & $0.863 \mathrm{~h}^{-1}$ & {$[0.24,1.38]$} & Augustin et al. (2005) \\
$K_{i}, K_{p}$ & carrying capacity & $10^{8} \mathrm{CFU}$ & {$\left[10^{5}, 10^{11}\right]$} & Lecuit (2001) \\
$\beta_{T}$ & killing rate & $10^{-3} \mathrm{~h}^{-1}$ & {$\left[10^{-6}, 10^{0}\right]$} & this study \\
$\alpha$ & saturating factor & $10^{-7} \mathrm{CFU}^{-1}$ & {$\left[10^{-10}, 10^{-4}\right]$} & this study \\
$C$ & gastric pressure & $0.10 \mathrm{CFU}^{-1}$ & {$[0,1.0]$} & this study \\
$q$ & T-cell proliferation rate & $10^{-3} \mathrm{~h}^{-1} \mathrm{CFU}^{-1}$ & {$\left[10^{-10}, 10^{0}\right]$} & this study \\
$m$ & mobility rate & $0.20 \mathrm{CFU}^{-1}$ & {$[0,1.0]$} & this study \\
$\mu$ & death rate of T-cell & $0.01 \mathrm{~h}^{-1}$ & {$\left[10^{-4}, 10^{0}\right]$} & Gorman et al. (2014) \\
\hline
\end{tabular}
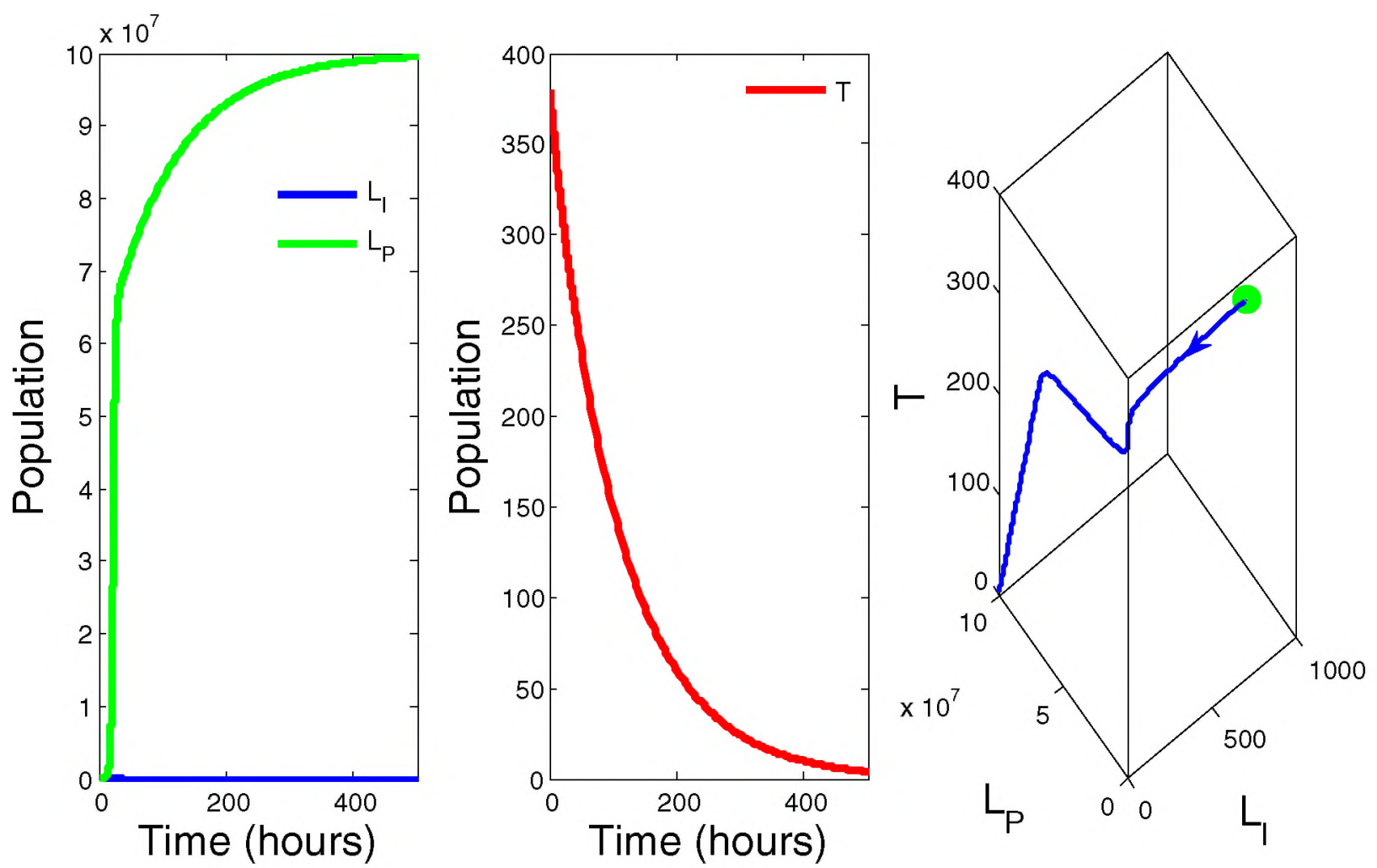

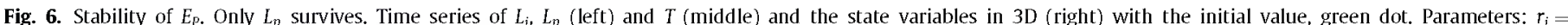

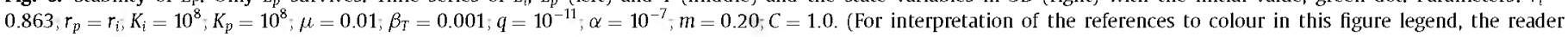
is referred to the web version of this article.)

the dynamics of the model evolve through the variation of these parameters. The parameter $C$, regarding the innate immune cells and intestinal molecules, has a significant effect on the survival of $L$. monocytogenes as described by the model analysis and by the earlier model in Rahman et al. (2016). To observe the effect of $C$ on the existence and stability of $E_{*}$, we generate a bifurcation diagram in Fig. 7. Initially, a single positive equilibrium exists which is stable. As $C$ increases and reaches a critical value, another equilibrium emerges which destabilizes both equilibria. Mathematically, this means that system trajectories will approach one of the remaining stable equilibria. In particular, this indicates that for larger values of $C$, the $L$. monocytogenes population in the small intestine cannot persist.

Next, we consider the growth parameter $r_{i}$. Fig. 8 shows a somewhat opposite trend than the bifurcation associated with $C$. When $r_{i}$ crosses through a critical value, 0.55 , both equilibria appear one of which is stable. As $r_{i}$ increases further, the unstable equilibria disappears while the stable equilibrium continues to exist. Note that as the $L_{i}$ population increases through $r_{i}$, the $L_{p}$ population decreases as can be seen in Fig. 9 and from the Eq. (3.5).

\section{Discussion}

L. monocytogenes is an acute problem for the food industry as well as for public health. Understanding the key infection mechanisms of this pathogen is crucial for correctly prioritizing prevention tools to reduce the infection burden. A biologically informed within host model with a small number of parameters, varying in plausible ranges, can help to visualize the wide spectrum of the pathogenesis in the absence of extensive scientific experiments. This information is useful for designing scientific experiments and testing hypotheses.

We developed a novel mathematical model (Rahman et al., 2016) in order to demonstrate the dose-response relationship of L. monocytogenes with mechanistic approach. The model (Rahman et al., 2016) describes the pathogen host interaction in the initial pathway, mouth to small intestine, of L. monocytogenes to demonstrate the potential of the pathogen for causing an infection. This paper extends the model in Rahman et al. (2016) to describe the complete pathway of $L$. monocytogenes with-in the host. The current model (2.1) highlights the immune response of the host, failure of which can cause multiple organ infection leading to Listeriosis (Cossart and Toledo-Arana, 2008). 


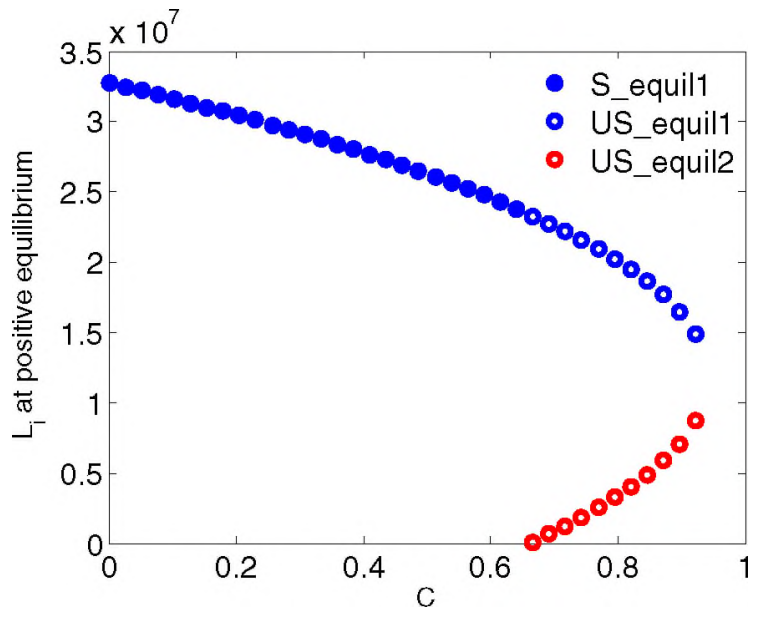

Fig. 7. Bifurcation of existence and stability of $E_{*}$ with respect to C. Initially, a single equilibrium exists and is stable. As $C$ increases and reaches a critical value, another equilibrium emerges which destabilize both equilibria. In the later case, when both positive equilibria become unstable, the trajectories will approach one of the remaining stable equilibria. Other parameters: $r_{i}=0.863 ; r_{p}=r_{i} ; K_{i}=10^{8} ; K_{p}=$ $10^{8} ; \mu=0.01 ; \beta_{T}=0.001 ; q=10^{-10} ; \alpha=10^{-7} ; m=0.20$.

The results from the model analysis provide thresholds that depend on model parameters. These thresholds determine the existence of infection 'states', indicating under which parameter regimes the dynamics of the infection will switch from one state to other. By the existence of 'state' we mean whether $L_{i}$ (the $L$. monocytogenes population in the small intestine) or $L_{p}$ (the $L$. monocytogenes population in the lamina propria) or both can survive long term. Steady state analysis gives the long term behaviour of the model solutions, indicating the trajectories of the state variables. More precisely, it can demonstrate whether a state variable (pathogen) will be eliminated from or remain in the host. It also provides the information regarding the possible pathogen load, if

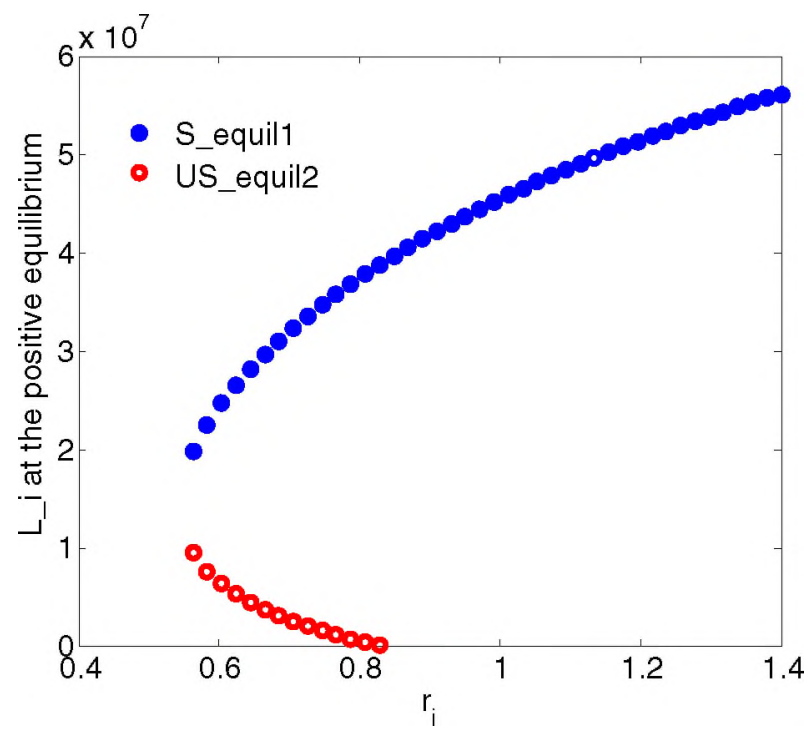

Fig. 8. Bifurcation of existence and stability of $E_{*}$ with respect to $r_{i}$. When $r_{i}$ crosses through a critical point, 0.55 , both equilibria appear one of which is stable. As $r_{i}$ increases further, the unstable equilibria disappears while the stable equilibrium continue to exist. Other parameters: $r_{p}=0.53, K=10^{8} ; K_{p}=10^{8} ; \mu=$ $0.01 ; \beta_{T}=0.001 ; q=10^{-10} ; \alpha=10^{-7} ; m=0.002 ; C=0.83$.

it survives. Such information is important for establishing correlations between symptoms of the infected host and infection states.

We identify the ranges of key parameters, through bifurcation analysis, for which $L$. monocytogenes can be sustained at high or low levels in the host or could be eliminated from the host. As shown in Fig. 7 , as the parameter $C$ increases, the positive equilibrium $E_{*}$ ceases to exist. On the other hand, the positive equilibrium $E_{*}$ appears when the growth rate $r_{i}$ increases to a certain threshold (Fig. 8). The increase of $r_{i}$ helps increase the $L_{i}$ at a larger scale. This behaviour is well justified. As $C$ increases, the $L_{i}$ popu-
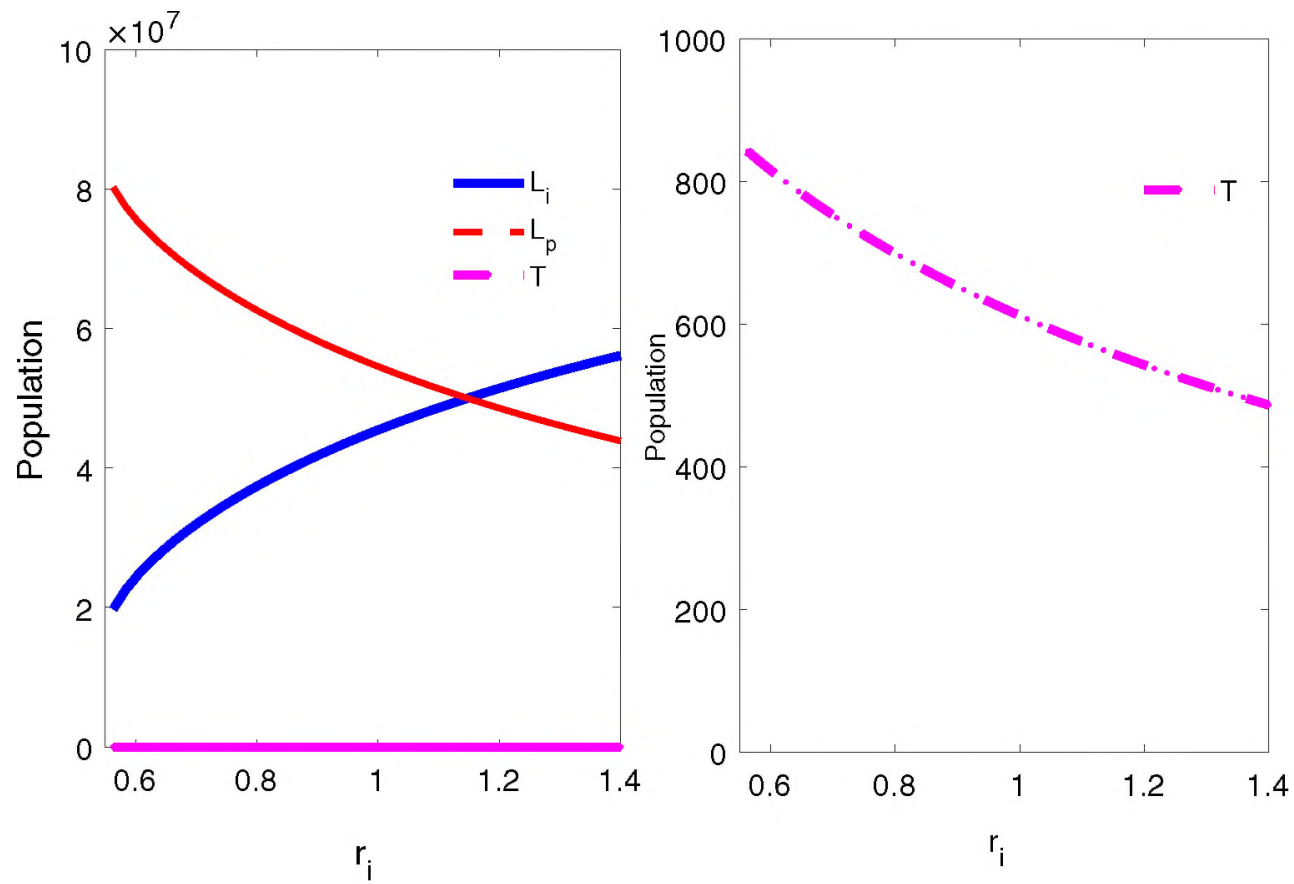

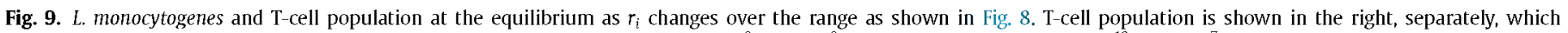
is not visible in the left figure due to scaling. Other parameters: $r_{p}=0.53, K_{i}=10^{8} ; K_{p}=10^{8} ; \mu=0.01 ; \beta_{T}=0.001 ; q=10^{-10}, \alpha=10^{-7} ; m=0.002 ; C=0.83$. 

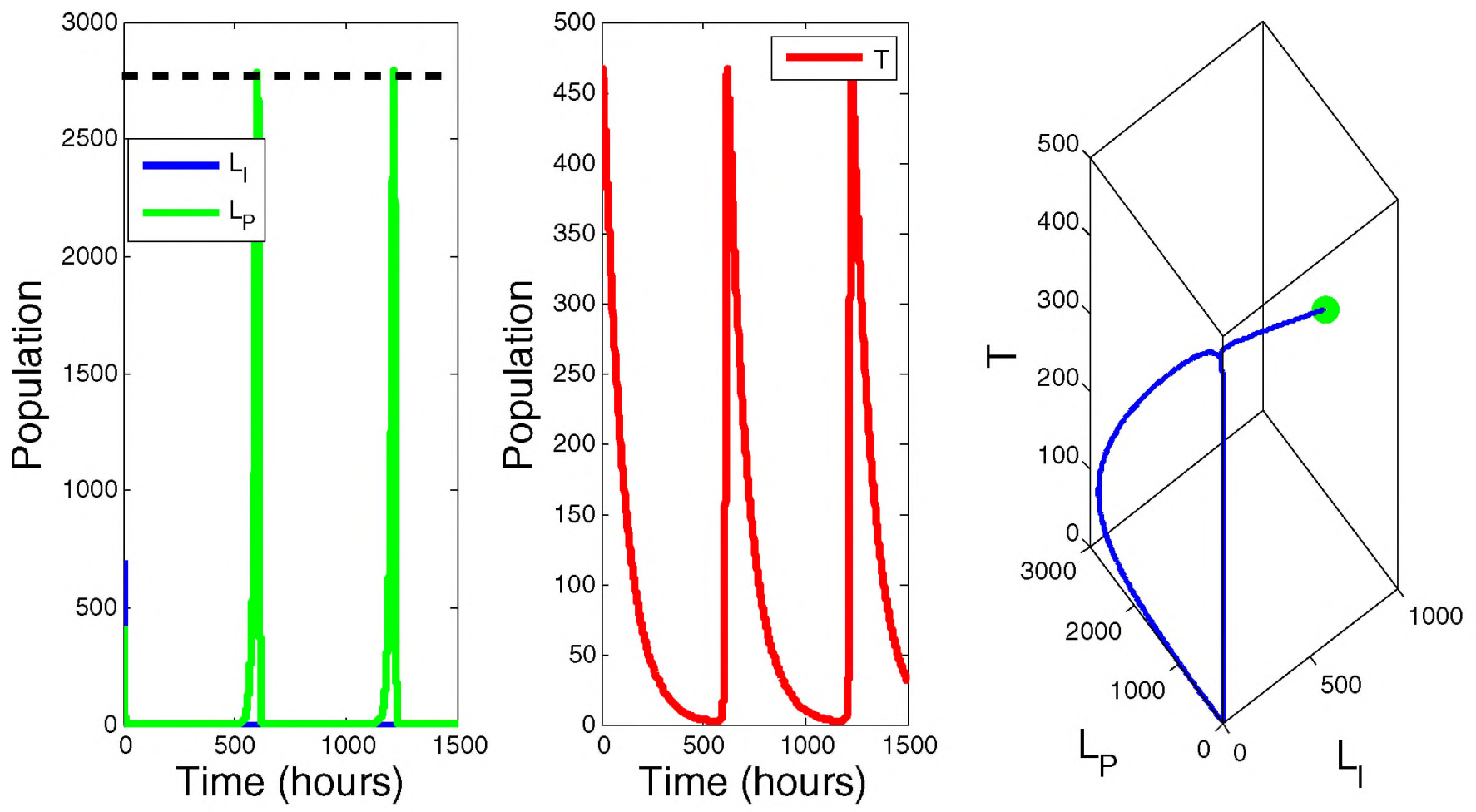

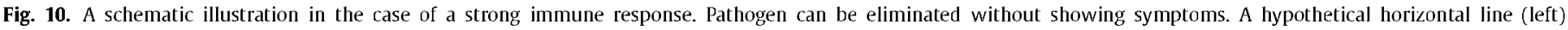

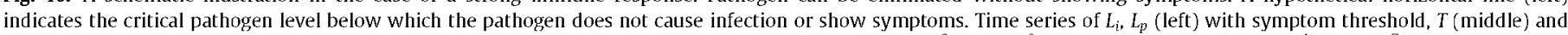

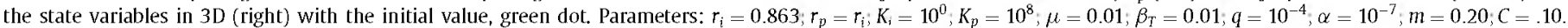
(For interpretation of the references to colour in this figure legend, the reader is referred to the web version of this article.)

lation in the small intestine cannot be sustained. Similarly, when the growth rate increases it helps to sustain the pathogen in both locations: small intestine and lamina propria.

The most important application of this model is to identify the pathogen-host interaction and to quantify the immune response of the host against pathogens. The adaptive immune system equipped with T-cells responds to any sustained infection that cannot be eliminated by the innate immune system. The strength of the immune response is related to how rapidly a pathogen specific T-cell is generated by the host. This is measured by the parameter $q$. A small value of $q$, related to immuno-compromised individuals, may not limit the pathogen load within the tolerance level which in turn could lead to a severe infection or death. Figs. 2-5 demonstrate the significance of $q$ in order to control $L$. monocytogenes in a host. The pathogen can survive in both locations of a host with a very weak immune system (Fig. 2). With a larger $q$, the pathogen can be eliminated from the host or limited to a low level.

\subsection{Application of the model to public health}

The model can be applied to identify the growth potential of $L$. monocytogenes in a host and the immune response of the host relative to the pathogen presence. In particular, the model can be utilized as a tool to identify the time period from exposure to disease onset by connecting pathogen levels to disease symptoms. The $L$. monocytogenes specific T-cells could also be a good indicator for disease onset. Informed by specific experiments, our model can provide a means to estimate the correlation among T-cell levels, the pathogen load and clinical symptoms. Such information would be invaluable for advancing clinical effectiveness on the individual level and risk analysis at the population scale.

The model can also be used to explain how the pathogen can survive in a host for a long time without showing symptoms as depicted in Fig. 10. If the pathogen load remains below a threshold, the immune system may tolerate the pathogens and allow them to survive for a longer period in an asymptomatic state. Due to the assumption of constant death rate of T-cells, model allows for the possibility that pathogen levels oscillate with the T-cell population as shown in the Fig. 10. However, this is an artifact of the model as the T-cells population would not go down to zero necessarily (Lecuit, 2001; Pamer, 2004). In fact, not all T-cells die out, some memory T-cells remain in the immune system to fight any recurrent infections (Pamer, 2004). For simplicity, we do not consider memory cells in the model rather we focus on demonstrating the key infection events in the pathogen-host interaction.

\subsection{Future directions}

Despite the illustration of potential use of model (2.1) for understanding the with-in host infection of $L$. monocytogenes, our formulation has some limitations. The role of the innate immune cells on the dynamics (e.g. pathogen levels) is not included in the model under the assumption that such an effect is negligible as compared to T-cell responses. Mathematically speaking, this is reflected in the fact that we hold $C$ constant in model (2.1), whereas $T$ is able to grow in time. However, to eliminate the pathogens from the host the role of innate immune cell could be important (Carrero and Unanue, 2007), particularly, when the pathogen level remains low. The memory cells of the adaptive immunity originated from the earlier infections could also play a significant role (Finelli et al., 1999; Pamer, 2004; Seaman et al., 2000) to prevent the current infection. Model (2.1) could be adapted to include the dynamics of memory cells.

Our model also operates under the assumption that death rate of T-cells is linear. However, the activated T-cells may not start dying (apoptosis) until they receive signals from specific death receptors, typically occurring when the majority of the infection is over (Crispe, 1999; Seaman et al., 2000). The function of death receptors 
and the cell-cycle mechanism, when incorporated in the model, could provide a subtle but crucial result to explain why individuals at either end of the age spectrum succumb to infections more easily. In terms of the model (2.1), $\mu$ (death rate of T-cells) should be an appropriate function of time. Furthermore, $L$. monocytogenes may not start replicating in the host right away, rather may remain in a dormant state for a long time without causing infection. In fact, evidence shows that up to $10 \%$ healthy humans could be intestinal carriers of L. monocytogenes (Henning and Cutter, 2001). Inclusion of the delay on the growth function of $L$. monocytogenes may reveal some interesting and complex dynamics. Future work may include the role of stochastic effects which, for oscillatory behaviour, eventually will lead to extinction of bacteria from the host. One may be interested to study the possibility of pattern formation by the bacteria in a spatially extended system (Gray and Scott, 1984).

\subsection{Final notes}

We developed and studied a with-in host dynamic model for $L$. monocytogenes. The model could be easily generalized to any bacterial infection with minor modifications. Like any other models, the applicability of our model depends on further testing with scientific data.

\section{Acknowledgements}

Daniel Munther acknowledges support from Cleveland State University startup funding (STARTUP42). Ashrafur Rahman's postdoctoral fellowship is supported by a research contract from the Public H eath Agency of Canada and by the NSERC CREATE project Advanced Disaster, Emergency and Rapid Response Simulations. Jianhong Wu's research has been funded by the Natural Sciences and Engineering Research Council of Canada and by the Canada Research Chairs program.

\section{References}

Artis, D., 2008. Epithelial-cell recognition of commensal bacteria and maintenance of immune homeostasis in the gut.. Nat. Rev. Immunol. 8 (6), 411-420. doi:10. $1038 / 11$ ri2316.

Augustin, J.C., Zuliani, V., Cornu, M., Guillier, L, 2005. Growth rate and growth probability of Listeria monocytogenes in dairy, meat and seafood products in suboptimal conditions. J. Appl. Microbiol. 99 (5), 1019-1042. doi:10.1111/j.1365-2672. 2005.02710.x.

Banfi, E., Cinco, M., Zabucchi, $G_{\text {。, }}$ 1986. Phagocytosis of campylobacter jejuni and c, coli by peritoneal macrophages. J. Gen. Microbiol. 132 (Pt 8), 2409-2412.

Blanco-Lizarazo, C.M., Sotelo-Díaz, I., Llorente-Bousquets, A., 2016. In vitro modelling of simultaneous interactions of Listeria monocytogenes, Lactobacillus sakei, and Staphylococcus carnosus. Food Sci. Biotechnol. 25 (1), 341-348. doi:10.100\%/ s10068-016-0048-0.

Buchanan, R.L., Gorris, L.G.M., Hayman, M.M., Jackson, T.C., Whiting, R.C., 201\% A review of Listeria monocytogenes: An update on outbreaks, virulence, doseresponse, ecology, and risk assessments. Food Control 75, 1-13. doi:10.1016/j. foodcont.2016.12.016.

Carrero, J.A., Unanue, E.R., 200\% Impact of lymphocyte apoptosis on the innate immune stages of infection. Immunol. Res. 38 (1-3), 333-341. doi:10.100\%/ s12026-007-0017-z.

CDC, 2018. Listeria Outbreaks. Centers for Disease Control and Prevention, https: //www.cdc.gov/listeria/outbreaks/index.html, accessed May16, 2018.

Cossart, P., 2011. Illuminating the landscape of host-pathogen interactions with the bacterium Listeria monocytogenes.. Proc. Natl. Acad. Sci. U.S.A. 108 (49), 19484 19491. doi:10.1073/pnas.1112371108.

Cossart, P., Toledo-Arana, A., 2008. Listeria monocytogenes, a unique model in in fection biology: an overview. Microbes Infect. 10 (9), 1041-1050. doi:10.1016/j. micinf.2008.0\% 043 .

Crispe, I.N., 1999. Death and destruction of activated T lymphocytes.. Immunol. Res. 19 (2-3), 143-157. doi: 10.1007/BF02786483.

Falk, L.E., Fader, K.A., Cui, D.S., Totton, S.C., Fazil, A.M., Lammerding, A.M., Smith, B.A., 2016. Comparing listeriosis risks in at-risk populations using a userfriendly quantitative microbial risk assessment tool and epidemiological data. Epidemiol. Infect. 144 (13), 2743-2758, doi:10.1017/S0950268816000327.
FAO/WHO, 2004. Risk assessment of Listeria monocytogenes in ready-to-eat foods,www.fao.org/3/a-y5394e.pdf, accessed May 16, 2018. Technical Report. Food Safety Department, World Health Organization. Geneva.

Farber, J.M., Ross, W.H., Harwig, J., 1996. Health risk assessment of Listeria monocytogenes in Canada. Int. J. Food Microbiol. 30 (1-2), 145-156. doi:10.1016/ 0168-1605(96)01107-5.

Finelli, A., Kerksiek, K.M., Allen, S.E., Marshall, N., Mercado, R., Pilip, I., Busch, D.H. Pamer, E.G., 1999. MHC Class I Restricted T Cell Responses to Listeria monocytogenes, an Intracellular Bacterial Pathogen Listeria monocytogenes MHC tetramers Antigen presentation MHC class Ib molecules Cytolytic ' l lymphocytes Intracellular bacteria Antimicrobial immunity. Immunol. Res. 19 (2-3), 211-223.

Foodnet, 2017. National Enteric Disease Surveillance: The Listeria Initiative, https: //www.cdc.gov/listeria/pdf/ListeriaInitiativeoverview_508.pdf, accessed May 18, 2018. CDC.

Gorman, J.V., Starbeck-Miller, G., Pham, N.-L.L., Iraver, G.L., Rothman, P.B., Harty, J.I., Colgan, J.D., 2014. 'lim-3 directly enhances CD8 T cell responses to acute $L$ is teria monocytogenes infection.. J. Immunol. 192 (7), 3133-3142. doi:10.4049/ jimmunol.1302290.

Gray, P., Scott, S.K., 1984. Autocatalytic reactions in the isothermal, continuous stirred tank reactor. Oscillations and instabilities in the system $\mathrm{A}+2 \mathrm{~B}$ ? $3 \mathrm{~B}$; B ? C. Chem. Eng. Sci. 39 (6), 108/-1097. doi:10.1016/0009-2509(84)8/01/-\%.

Haas, C.N., Thayyar-Madabushi, A., Rose, J.B., Gerba, C.P., 1999. Development and validation of dose-Response relationship for Listeria monocytogenes. Quant. Micro biol. 1 (1), 89-102.

Henning, W., Cutter, C., 2001. Controlling Listeria Monocytogenes in Small and Very Small Meat and Poultry Plants. Technical Report. Food Safety and Inspection Service, USDA.

Hethcote, H.W., 2000. The mathematics of infectious diseases. SIAM Rev. 42 (4) 599-653. doi: $10.113 / /$ S00361445003/190\%.

Lecuit, M., 2001. A transgenic model for listeriosis: role of internalin in crossing the intestinal barrier. Science 292 (5522), 1722-1725. doi:10.1126/science 1059852

Mead, P., Slutsker, L., Dietz, V., McCaig, L., Bresee, J., Shapiro, C., Griffin, P., Tauxe, R., 1999. Food related illness and death in the United States.. Emerging Infect. Dis. $5(5), 60 \%-625$.

on Immunization (NACI), N.A.C., 2013. Canada communicable disease report update on the use of quadrivalent 39 (January), 1-40.

Pamer, E.G., 2004. Immune responses to Listeria monocytogenes. Nat. Rev. Immunol. $4(10), 812-823$, doi: $10.1038 /$ nri1461.

PHAC, 2018. Public health notices. Public Health Agency of Canada, https: //www.canada,ca/en/public-health/services/public-health-notices.html, accessed May 16, 2018.

Pouillot, R., Klontz, K.C., Chen, Y., Burall, L.S., Macarisin, D., Doyle, M., Bally, K.M. Strain, E., Datta, A.R., Hammack, T.S., Van Doren, J.M., 2016. Infectious dose of Listeria monocytogenes in outbreak linked to ice cream, United States, 2015. Emerging Infect. Dis. 22 (12), 2113-2119, doi:10.3201/eid2212.160165.

Rahman, A., Munther, D., Fazil, A., Smith, B., Wu, J., 2016. Unraveling the dose response puzzle of $L$ monocytogenes: a mechanistic approach. Infectious Disease Modelling 1 (1), 101-114. doi:10.1016/j.idm.2016.09.001.

Ribet, D., Cossart, P., 2015. How bacterial pathogens colonize their hosts and invade deeper tissues. Microbes Infect. 1/ (3), 173-183. doi:10.1016/j.micinf.2015. 01.004 .

Roulo, R.M. Fishburn, J.D., Amosu, M, Etchison, A.R, Smith, M.A, 2014 . Dose response of Listeria monocytogenes invasion, fetal morbidity, and fetal mortality after oral challenge in pregnant and nonpregnant mongolian gerbils. Infect. Immun. 82 (11), 4834-4841. doi:10.1128/IAI.01514- 14.

Schvartzman, M.S., Gonzalez-Barron, U., Butler, F., Jordan, K., 2014. Modeling the growth of Listeria monocytogenes on the surface of smear- or mold-ripened cheese. Front. Cell. Infect. Microbiol. 4 (July), 1-9. doi:10.3389/fcimb.2014 00090 .

Seaman, M.S., Wang, C.R., Forman, J., 2000. MHC class Ib-restricted CTL provide protection against primary and secondary Listeria monocytogenes infection. J. Im munol. 165 (9), 5192-5201. doi:10.4049/jimmunol.165.9.5192.

Smith, H.L., Waltman, P., 1995. The theory of the chemostat : Dynamics of microbial competition doi: $10.230 \% / 2405002$.

Smith, M.A., Takeuchi, K., Anderson, G., Ware, G.O., Mcclure, H.M., Raybourne, R.B., Mytle, N., Doyle, M.P., 2008. Dose-response model for Listeria monocytogenesinduced stillbirths in nonhuman primates. Infect. Immun. 76 (2), 726-731. doi:10.1128/IAI.01366-06.

Stafford, M.A., Corey, L., Cao, Y., Daar, E.S., Ho, D.D., Perelson, A.S., 2000. Modeling plasma virus concentration during primary HIV infection. J. Theor. Biol. 203 (3), 285-301. doi:10.1006/jtbi.2000.1076.

Stecher, B., Hardt, W.-D., 2011. Mechanisms controlling pathogen colonization of the gut Curr Opin. Microbiol, 14 (1), 82-91, doi:10.1016/j.mib.2010,10,003.

Văzquez-boland, J., Kuhn, M., Berche, P., Chakraborty, T., Domi, G., González-zorn, B, Wehland, J., 2001. Listeria pathogenesis and molecular virulence determinants listeria pathogenesis and molecular virulence determinants. Clin. Microbiol. Rev. 14 (3), 584-640. doi:10.1128/CMR.14.3.584. 